\title{
Energy saving using location aware sensor networks
}

\author{
Eoin Hurrell \\ CLARITY: Centre for Sensor Web Technologies \\ School of Computing, Dublin City University \\ Glasnevin, Dublin 9, Ireland \\ Eoin.Hurrell@computing.dcu.ie
}

\author{
Alan F. Smeaton \\ CLARITY: Centre for Sensor Web Technologies \\ School of Computing, Dublin City University \\ Glasnevin, Dublin 9, Ireland \\ Alan.Smeaton@dcu.ie
}

\begin{abstract}
This work reported in this paper exploits a network of inexpensive wireless sensors to improve the energy efficiency of desktop computer usage in a major business or campus environment. Computer users frequently leave their desktop machines permanently switched on or in sleep mode when finished using them, in order to quickly resume work when returning. This results in unnecessary power consumption for a relatively small return in terms of user convenience. Eliminating this unnecessary power consumption without sacrificing user convenience is what our research achieves. The novelty of this work is that it achieves this goal using ambient sensors to establish user context.
\end{abstract}

\section{Keywords-Energy saving;sensor network;power management}

\section{INTRODUCTION}

There has been much recent research into electricity monitoring from a range of research communities, including Toller et al. who investigated the energy usage patterns of the Swedish building and real estate management sector [5], and Bristow et al. who exploited current data-rich energy monitoring technologies to analyze hour-by-hour greenhouse gas emissions for a low-energy condominium design [4]. In a business context the problem of electricity usage is particularly driven by a growing and sometimes excessive expense of maintaining one of the most common office devices: the desktop computer. The ubiquity of computers in the office has presented an energy conservation challenge. Computer manufacturers continue to design more 'green' energy-efficient machines and many countries legislate for a carbon tax on the emissions caused by heavy-duty energy usage. Additionally energy providers are installing smart meters in homes and businesses order to inform users of their usage. This feedback has been shown to increase energy-usage awareness, with a typical saving of $5-15 \%[6,9]$ or $5-12 \%$ [8] and a summary of the work done in this area can be found in Hazas et al. in [10].

A less frequently explored influencer of energy consumption is the human element. While the focus has been on informing users of energy usage, with good reason as it makes them more energy-conscious [3], our work examines the effect of automating the power-management of a device (the desktop computer) for a set of users (workers in an office) based on establishing the users' contexts. The aim is to support existing user behaviour as much as possible rather than challenge it as previous research has done [6]. This in turn will lead to a greater saving without the need to enforce a policy

The authors gratefully acknowledge support from Science Foundation Ireland under grant 07/CE/I147, CLARITY CSET based on user understanding, and a cost of running in line with actual computer usage.

In the long term this avoids the damage to the environment resulting from leading unsustainable lifestyles based on wasteful energy consumption. It is a fact of life that computers are now a key component of business, and we must adjust our lifestyles to account for this, or develop systems to adjust their operation for us. Here we aim to show the benefits of using a network of sensors to aid computer users and businesses in their goals and to support existing lifestyles and habits while being environmentally aware.

Wireless sensor networks are frequently used in environmentally aware settings, including applications such as remote water-quality monitoring and landfill carbon dioxide tracking. The technology allows for locationally or even methodologically disparate sensor modules to leverage their unique context in a wider setting, and inform a bigger picture than a single sensor deployment ever could. This paper explores their use in conserving energy in an office setting. Our application for sensor networks was inspired from multiple studies that variously concluded that up to half of office workers [1] (44\% in Germany for example, [2]) leave their desktop computers on overnight and over weekends, in order to begin work without the delay of a computer boot-up in the morning.

Ideally machines would be truly instantly on, and effectively as energy-efficient while in standby as when shut down, and work is being done towards that end, but the human side of the equation can equally be examined and human behaviour adjusted or changed to save even more energy. The interaction between user and device happens in a given context, and establishing that context could one day enable us to automate complex power management strategies like powering up everything we need when we come into a room, an arrangement to have everything just ready for you when you get to it without hassle or worry. It is important to attempt to optimize for the human element in this way, and a sensor web can place the user in just such a context.

Our aim is to reduce high energy expenditure on a large scale. Our system maintains awareness of the presence of users within their usual workplace location and can determine when users arrive and depart. It can then cut down the amount of time computers are powered on and in idle or sleep mode by turning them completely off (zero watt power consumption) and then intelligently choosing when to power them up from 
off when their associated users return to the building. Desktop computers can be remotely triggered in response to a user being sensed by the network in a situation appropriate for action, for example after returning to the office in the morning or coming back from lunch. The system takes into account the whereabouts of the user and the time since they have been seen when activating their machine. Users arrive at their desk to a powered on computer with no inconvenience or delay.

\section{APPROACH}

This paper outlines a new and novel approach to the use of wireless sensor networks for energy saving, by using them to detect users in a space and control their energy usage based on their contexts. In its current implementation this is ideal for office computer management, but the general concepts introduced could easily scale to home use with the correct sensor deployment.

This approach is designed to leverage the fact that our energy-conservation intentions can be foiled by our needs. In order to have a working computer ready to use in the morning it is easier to leave it on overnight than wait for it to boot up. Any hindrance in usability without feedback or visible personal improvement will stop us from making savings in energy use and $\mathrm{CO} 2$ emissions.

Rather than shutting down computers completely on a schedule, which would hinder users and not accurately mirror activity resulting in sub-optimal use, we attempt to understand the user's activities in a space and work to fulfill their needs. In our approach the mobile phone's Bluetooth, a technology that exists in all modern handsets, uniquely identifies the user. While this is a well-accepted personal identifier, application developers have leveraged this for security purposes such as locking a computer or even physical locks; this has never been used in conjunction with a web of sensors to contextualize the data which can be gathered on beluetooth presence. The contextual web that drives this energy management has many other uses, some of which are touched on later.

Given the problem of inefficient energy usage due to user requirements it is desirable to control the power flow of an office building based on the context of the users present in the building. We have therefore created a new application for wireless sensor networks in the energy-saving domain, using network-detected events to trigger energy-saving activities, independent of user control. This approach is designed to be supportive of user lifestyle in order to optimize their desktop computer's power consumption based on their actual usage, with little overhead in order to give them an uninterrupted experience.

\section{METHODOLOGY}

Our system is designed to make use of the machines that exist in a physical network around us. Like the network distributed through a house for an alarm system this technology could in future be deployed discreetly in the home for its purpose, here we have outlined a network that detects users' mobile phones and uses them as a form of identification, that tells the system where, within the physically distributed network, a user is. Armed with this location the system can infer the best course of power-saving action. Our own implementation makes use of Apple MAC Minis as inexpensive Bluetooth sensors, networked together.

The system is comprised of an application running on a central server and multiple Bluetooth sensors distributed in areas deemed to be important. Each of these sensors sweeps the area every ten seconds for Bluetooth devices. This information stored on the devices as log files, detailing which devices was seen at that time. The server application then scrapes the data from the sensor nodes, meaning all information is fed to one central location that keeps track of all devices.

The Mac mini computers used as sensors for this deployment use $183 \mathrm{Kw} / \mathrm{H}$ annually maximally, significantly less than an average desktop computer, if left constantly on. This can be reduced with intelligent power managing (i.e. scheduling them to turn off after everyone leaves and on before anyone arrives) to $31 \mathrm{Kw} / \mathrm{H} \mathrm{[1]}$. These devices record a snapshot of all the Bluetooth devices they see every ten seconds, with unique MAC addresses, given names and time all recorded. The server takes these logs and maintains a list of all seen devices so users can link their mobile phone or other detectable Bluetooth device and their desktop computer. This provides a useful portal to show who is in the various sensorequipped areas.

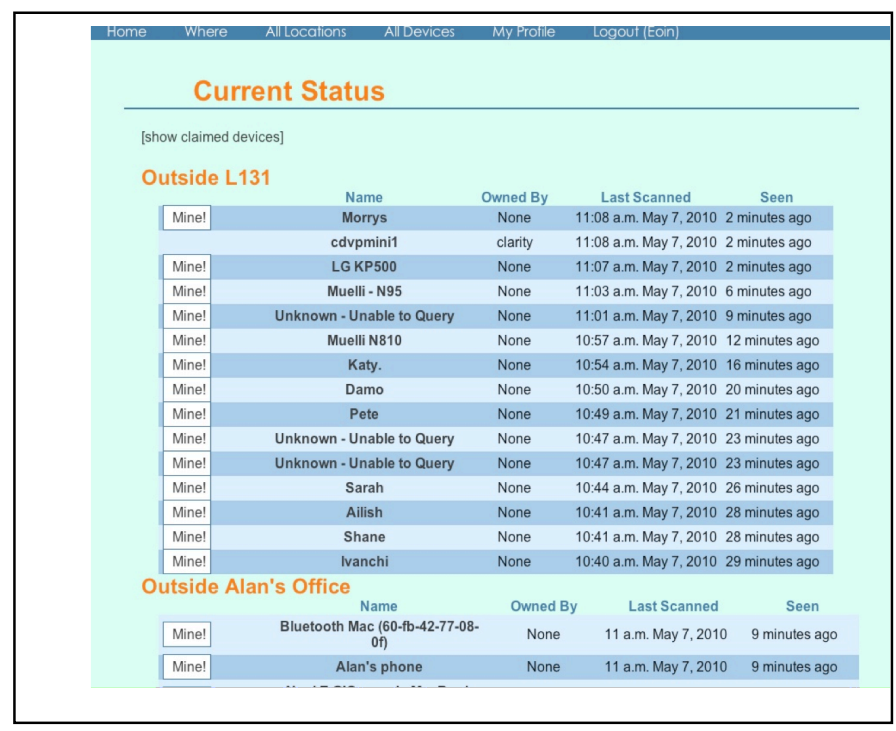

Figure 1. Server Interface

Key to our method is its use of Wake On LAN (WOL) technology, designed for system administrators, that allows a computer to be activated from complete shutdown, as well as from sleep or hibernation. This is preferable to putting computers in stand-by low-power mode, a compromise that uses $20 \%$ of a machine's full power continually in order to switch on with improved speed. This WOL protocol uses Ethernet connections and as such is restricted, but with work being done on network-controlled plugs the possible devices would be limitless, though we confine the study here to desktop computers. The implications of this are much greater 
energy savings than power management techniques such as schedule hibernations, with the added flexibility of corresponding to actual usage.

Since not only the current position of the user is stored but also their historic movements through the sensor space the network can, if needed, predict when someone will need their computer, even if they are not detected. This enables a more complex modeling of user energy consumption in future, especially with a well-blanketed area covered by sensors. Basic modeling is done in order to make decisions on whether to attempt to turn on a machine for example, if the user is seen entering the building 5 minutes after they left then their machine will still be on for them, and it makes no sense to attempt to power it up. We can also use the statistics to make assumptions about when a user will be around based on the number of times they are seen in an average day.

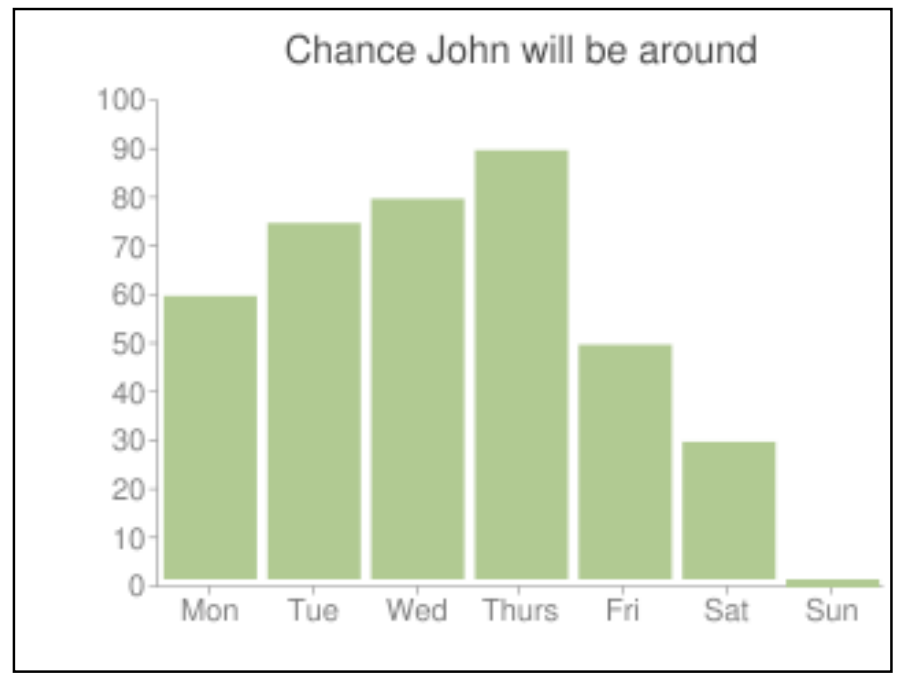

Figure 2. Example estimate graph of when a user will be around

\section{ANALYSIS}

In our trial deployment of this system we set up a network of sensors designed to intelligently map user movement in a two-storey building on a University campus. In order to adequately cover the area we used sensors placed at the entrance, first floor and second floor respectively. These three MAC minis were already in use as newsfeed displays, an example of the embedded computing frequently seen in an office space. Using these we logged 3,500 unique Bluetooth devices over a 3 -month period.

\section{EVALUATION 1 - SINGLE COMPUTER}

In order to test the comparative energy saving of this method we measured the power usage at the plug of two desktop computers, over two weeks. During the first week both machines were used as normal, and during the second the sensor network controlled them. The users were instructed not alter their behaviour or usage in any way during the tests in order to produce accurate results. Their energy usage was averaged to produce the following results:

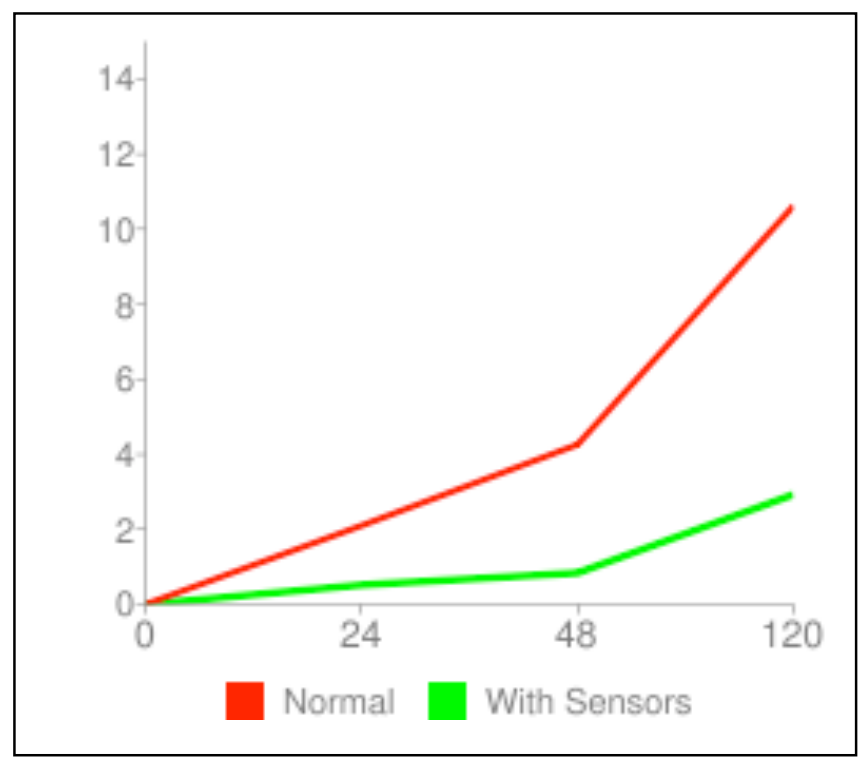

Figure 3. Comparison of energy usage

TABLE I. KW/H SAVINGS IN TIME

\begin{tabular}{|c|c|c|c|}
\hline Time & Normal & $\begin{array}{c}\text { With } \\
\text { Sensors } \\
\end{array}$ & Savings \\
\hline 24 hours & 2.10 & 0.52 & $\begin{array}{c}1.58 \\
(75 \%)\end{array}$ \\
\hline 48 hours & 4.26 & 0.84 & $\begin{array}{c}3.42 \\
(80 \%)\end{array}$ \\
\hline 120 hours & 10.62 & 2.93 & $\begin{array}{c}7.62 \\
(72 \%)\end{array}$ \\
\hline
\end{tabular}

\section{EVALUATION 2 - OFFICE-WIDE}

Given this excellent level of energy saving, we examined the possible benefits that this energy saving would have in a real-world environment. Our real-world example is the recorded logins in a set of 3 computer laboratories used during a typical month by computing students in a major university. These areas are more high-traffic than offices filled with workers but provide real statistics on computer usage, in a manner that is indicative of users spending short periods on machines between classes and tutorials. In this environment all the machines are left on for users to pick and choose whichever one they favour, an inefficient practice that exists in order to accommodate human behaviour. Each lab has 50 desktop machines, which vary in make from older models, which use on average 108 watts to newer that use a more energy efficient 48 watts while on. We collected data on 2,588 logins for 139 unique machines. The average time between one login and the next was 1 hour, 7 minutes and 58 seconds. For an evaluation we assume that after that time the currently logged in user has logged out, and the computer should shut down. Using these numbers results in a total of 1,802 hours, 54 minutes and 49 seconds of idle full-powered computers, on top of the 11 computers that are powered but never used $(5,280$ hours 
assuming they use basic power management to turn on at 6am and off at $10 \mathrm{pm}$ ). In just one month there are nearly 7,083 hours worth of computers fully powered when they need not be, at a cost of between 764.96 and $339.98 \mathrm{Kw} / \mathrm{H}$, or between 417.07 and 185.36 tonnes of carbon. In monetary terms, at a rate of $14 \mathrm{c}$ per Kwh, the saving is between $€ 47$ and $€ 107$ per month. The following table details these savings against the power usage of a schedule.

TABLE II. OFFICE-WIDE SAVINGS

\begin{tabular}{|l|c|c|c|}
\hline Machines & $\begin{array}{l}\text { Scheduled } \\
(\mathbf{K w} / \boldsymbol{H})\end{array}$ & $\begin{array}{l}\text { Potential Saving } \\
(\mathbf{K w} / \boldsymbol{H})\end{array}$ & $\begin{array}{l}\text { Potential Saving } \\
\text { (Carbon) }\end{array}$ \\
\hline Older machines & 7776000 & 764.96 & 417.07 \\
\hline Newer machines & 3456000 & 339.98 & 185.36 \\
\hline
\end{tabular}

\section{CONCLUSIONS}

Presented here is a model for large-scale energy conservation in an enterprise environment, which makes possible a system of energy-use, optimized for personal activity without the need for action on the user's part. This shows cumulative savings against a traditional approach of letting the user manage their own power usage, even when the user is 'energy conscious', though larger deployments and tests could be done to explore lifestyle differences and produce more robust results. The sensor network accurately models usage and essentially pays for itself in deployments of even small numbers of computers. This experience of supporting user behaviour while striving for energy-efficiency shows valuable lessons that may guide future discussion on the topic (like the topic of eco-design as discussed in sessions at ACM CHI [3]).

Combined with network-enabled actuating power supplies that are on the market currently, the methods outlined here could be expanded to control entire rooms based on the activities of users, for example 'powering up' the office's meeting room when a visitor arrives. Further work is being done to intelligently identify devices based on their energyusage signature, which could also be used in conjunction with our technology to model users and switch on specific devices based on their usage.

Energy savings are clear when using this system on a single computer, but for an environment with many machines managed intelligently with the sensor network, such as a large business or campus, the savings can be considerable. We have deployed a wireless sensor network in a typical office building that is fully automated to record all discovered Bluetooth devices. The system associates a user with their desktop computer in the office, and activates it based on the user's presence in the building. A survey conducted in 2008 [2] showed that $44 \%$ of Germany's computer-using workforce leaves their computers on when they leave their office. Assume an office building with 500 modern energy-saving computers, each assigned to a user, and working 5 days, 8 hours per day. If $44 \%$ of users leave their computer in sleep or idle mode, using our intelligent power management would save an average of up to $€ 542.41$ in just one month, which equates to almost 2 tonnes of carbon dioxide. From our own tests we have found that a conventional desktop computer could consume as much as five times less energy than when they are intelligently managed, many for the sole convenience of not having to wait for the machine to boot in the morning. Our work will examine the power-saving potential of the approach in a real-world officespace of 140 computers with users who spend short bursts of time with their computers during office hours.

As machines become more and more efficient in their energy consumption and move close to their optimum in terms of energy efficiency, human activity and human control of energy usage comes under ever-closer scrutiny. It is clear that our wasteful habits unfortunately prove very difficult to unlearn, especially if there is an associated inconvenience to us. Our research seeks to wrap technology around our habits as painlessly as possible, without any downsides, while reducing energy usage and carbon emissions on a large scale. The benefit to society is that computers are switched on only when they are needed, with no inconvenience or delay to the end user.

\section{REFERENCES}

[1] (http://dssw.co.uk/research/computer_energy_consumption.html) Review of Computer Energy Consumption and Potential Savings by Megan Bray.

[2] (http://www.1e.com/energycampaign/downloads/PC_EnergyReport2009 -UK.pdf) The Power to Save Money by Harris Interactive for $1 \mathrm{e}$

[3] Blevis, Eli (Session Chair). Home eco behavior. In CHI'10: Proceedings of the 28th international conference on Human factors in computing systems, New York, NY, USA, 2010. ACM.

[4] D. Bristow, R. Richman, A. Kirsh, C. A. Kennedy, and K. D. Pressnail. Hour-by-hour analysis for increased accuracy of greenhouse gas emissions for a low-energy condominium design. Industrial Ecology, 15(3):381-393, 2011.

[5] S. Toller, A. Wadeskog, G. Finnveden, T. Malmqvist, and A. Carlsson. Energy use and environmental impacts of the Swedish building and real estate management sector. Industrial Ecology, 15(3):394-404, 2011.

[6] Y. Strengers. Smart metering demand management programs: challenging the comfort and cleanliness habitus of households. In OZCHI '08: Proceedings of the 20th Australasian Conference on Computer-Human Interaction, pages 9-16, New York, NY, USA, 2008. ACM.

[7] S. Darby. The effectiveness of feedback on energy consumption. Technical report, Environmental Change Institute, University of Oxford, 2006.

[8] C. Fischer. Feedback on household electricity consumption: a tool for saving energy? Energy Efficiency, 1(1):79-104, 2008.

[9] D. Velte and A. Martin. Energy services: Reducing the energy consumption of residents by behavioural changes. Technical report, BewareE, 2009.

[10] M. Hazas, A. Friday, and J. Scott. Look back before leaping forward: Four decades of domestic energy inquiry. Pervasive Computing, IEEE, 10(1):13-19, jan.-march 2011. 